\title{
On the Possibility of Transplanting Mental States
}

\author{
$\underline{\text { Alexander Poltorak }}$ \\ Neuroenhancement Lab, LLC \\ APoltorak@NeuroenhancementLab.com
}

\begin{abstract}
Background: Brain-states, which correlate with specific cognitive states, may be monitored with non-invasive techniques such as EEG and MEG that indirectly measure cortical activity. These cortical signatures provide insight into the neuronal activity which has been used to identify abnormal cortical function in numerous neurological and psychiatric conditions. Further, the induction of entrained cortical rhythms via transcranial stimulation is effective in imbuing brain-states correlated with such cortical rhythms.

Objective/Hypothesis: Mental states have distinct neural correlates. It may be possible to induce a desired mental state by replicating these neural correlates.

Methods: EEG/MEG recording of brainwaves from a "donor" in a particular mental state provide a specific signature. This signature is then inverted, filtered, and applied via transcranial stimulation.

Conclusion: We propose that mental states may be transferred between subjects by acquiring an associated cortical signature from a donor which, following processing, may be applied to a recipient via transcranial stimulation.
\end{abstract}

Keywords: Mental state, brain-state, brainwave, brain entrainment, transcranial stimulation, transcranial electric stimulation, TES, transcranial direct current stimulation, transcranial alternating current stimulation, transcranial magnetic stimulation, TMS, tDCS, osc-tDCS, tACS, transcranial pulsed current stimulation (tPCS), Cranial Electrical Stimulation, transcranial Endogenous Sleep-Derived; tESD, EEG, MEG 
It has been suggested by Crick and Koch [1] (see also [2]) that every mental state is expressed through unique neural signals, such as frequency oscillations, that are correlated with mood, cognition and motor functions. We hypothesize that it may be possible to induce a desired mental state by replicating its neural correlates.

Functional neuroimaging, such as electroencephalography (EEG) or magnetoencephalography (MEG), can capture the neuronal activity of localized brain regions which correlate with distinct cognitive or behavioral states (mental states). EEG recordings have demonstrated, for example, that the pattern of brain activity changes during meditative acts, and frontal cortex EEG activity has been associated with emotion induction and regulation (Yu et al. [3]; Dennis and Solomon [4]). EEG recordings reflect ionic fluctuations resultant of neuronal communication in the cortex arising from dendritic depolarizations (Nunez and Srinivasan [5]). Alternatively, MEG measurements reflect intracellular ionic fluctuations, which similarly result from action potentials (Hämäläinen et al. [6]). In both cases, the output measures correlate with localized cortical activity.

These EEG or MEG signatures may be inverted in order to stimulate, rather than record, cortical activity. Specifically, transcranial electric stimulation (TES; Annarumma et al. [7]), including transcranial alternating current stimulation (tACS) and transcranial direct current stimulation (tDCS; Utz et al. [8]) are used to electrically stimulate cortical activity, while transcranial magnetic stimulation (TMS; see [9]) uses a precise magnetic field in order to achieve a similar endpoint of electric current control. Typical brain entrainment methods utilize a constant stimulus (e.g., tDCS) or a synthetic waveform, which may be a step function modulated on a direct current (such as "electrosleep" [10]), a sinusoid modulated on an oscillatory direct current (osctDCS; D’Atri et al. [11]), or a fixed frequency modulated on an alternating current (tACS; [12]). Helfrich et al. [13] utilized simultaneous tACS stimulation combined with EEG recordings to show 
that, when tACS was applied to the parieto-occipital lobe of the brain, alpha wave activity increased and became synchronized with the entrainment frequency.

Here, we propose the use of these techniques in order to record and subsequently induce specific brain states. Thus, EEG/MEG may be used to record mental states, which may then be applied via TES (tDCS, osc-tDCS, tACS) or TMS in order to replicate the cognitive-behavioral state of the "donor." We have previously investigated this technique in the domain of sleep [14].

Generally, we propose to investigate the notion of "transplanting" mental states including sleep, attention, and learning, as well as emotional valence. Attention-states in the brain are primarily the result of the cognitive process of suppressing the detection of erroneous stimuli. This cognitive state is associated with specific neuronal oscillations (Schroeder et al. [15]) which may be captured via EEG or MEG. The neural oscillations associated with attention have been shown to be disrupted in a number of conditions including epilepsy (Besle et al., [16]), dyslexia (Thomson et al. [17]; Leong et al. [18]; Soltész et al. [19]), and schizophrenia (Lakatos et al. [20]). Therefore, the acquisition of a brainwave signature during states of attention in a healthy "donor" may prove valuable when applied to a recipient exhibiting attention deficits associated with disrupted or otherwise irregular cortical oscillations. Previous research shows that memory functions are acutely sensitive to neural entrainment and may be disrupted via TMS (Hanslmayr et al. [21]) indicating the possibility of an inverse, positive entrainment of these oscillations.

Similarly, emotional arousal and valence are correlated with distinct cortical signatures observable through EEG (Allen et al. [22]). Previous data indicate that happiness resultant of musical experience, for instance, is associated with increased theta frequency oscillations in the left frontal cortical hemisphere (Rogenmoser et al. [23]). Cortical oscillations associated with negative affect conversely correlate with decreased theta frequency oscillations in this same region. Notably, aberrant cortical oscillations have been observed in a range of affective disorders including major 
depression (Van der Vinne et al. [24]). Indeed, the left frontal hemisphere exhibits disrupted cortical rhythms in patients diagnosed with major depression as compared to healthy controls (Nusslock et al. [25]). Similar data have highlighted cortical asymmetry of frontal lobe oscillations in post-traumatic stress disorder (PTSD; Meyer et al. [26]). Simple cortical entrainment via binaural beat stimulation has already proven adequate for inducing specific emotional states (Chaieb et al. [27]). More directly, cranial electrotherapy has been demonstrated as an efficacious treatment for depression, anxiety, and certain forms of insomnia (Kirsch et al. [28]). Certain forms of depression may, in fact, respond better to transcranial approaches, such as TMS, as has been demonstrated in early data on patients with treatment-resistant major depression (Rosenberg et al. [29]).

Thus, our proposed approach to "transplant" (transfer) mental states by replicating neural correlates of the donor's state in a recipient is founded on two main principles. First, a large body of literature has identified distinct, measurable cortical signatures associated with specific brainstates ranging from those defining the sleep/wake cycle to those underlying emotional experience. Second, TES and TMS have been repeatedly demonstrated as efficacious, safe means by which cortical rhythms may be entrained with a high degree of location-specificity. Together, these findings provide the basis for our hypothesis that mental states can be "transplanted" (transferred) and provide the means by which a cortical signature may be obtained via EEG or EMG associated with a desired mental state of a "donor" that may, in turn, be processed, inverted, and subsequently applied to a recipient in order to induce said cognitive state via cortical rhythm entrainment using tACS, TMS or other stimuli such as light or sound. Theoretical considerations suggest that this hypothesis is plausible and deserves experimental verification. Importantly, using cortical signatures acquired from a "donor," rather than a fixed-frequency or synthetic waveform applications as is currently typical for TES techniques, offers the distinct advantage of replicating multi-phasic, temporally dynamic signals which we believe are more likely to induce naturalistic 
mind-states holistically. This technique may, therefore, provide a novel approach to the noninvasive treatment of a variety of disorders whose current treatments are limited to pharmacotherapeutic interventions.

\section{Figure 1:}

Illustrative schematic of proposed transplantation of mental state. Electroencephalography (EEG) is used to register a cortical signature associated with a distinct mental state from a donor. This signal is digitally processed and inverted before being applied to a recipient via transcranial electric stimulation (TES).

\section{Acknowledgments:}

The author wishes to thank Prof. Marom Bikson for his encouragement and useful discussions.

\section{References}

[1] Crick F, Koch C. Towards a neurobiological theory of consciousness. Seminars in the Neurosciences, 1990; (2), 263-75.

[2] Rees G, Kreiman G, Koch C. Neural correlates of consciousness in humans. Nature Reviews. Neuroscience 2002;3(4),261-270. https://doi.org/10.1038/nrn783

[3] Yu X, Fumoto M, Nakatani Y, Sekiyama T, Kikuchi H, Seki Y, Sato-Suzuki I, Arita H. Activation of the anterior prefrontal cortex and serotonergic system is associated with improvements in mood and EEG changes induced by Zen meditation practice in novices. Int J of Psychophysiology 2011;80(2),103-111. https://doi.org/10.1016/j.ijpsycho.2011.02.004 
[4] Dennis TA, Solomon B. Frontal EEG and emotion regulation: electrocortical activity in response to emotional film clips is associated with reduced mood induction and attention interference effects. Biological Psychology 2010;85(3),456-464.

https://doi.org/10.1016/j.biopsycho.2010.09.008

[5] Nunez PL, Srinivasan R. Electric fields of the brain: the neurophysics of EEG (2 ed). Oxford: Oxford Univ. Press; 2006.

[6] Hämäläinen M, Hari R, Ilmoniemi RJ, Knuutila J, Lounasmaa OV. Magnetoencephalography - theory, instrumentation, and applications to noninvasive studies of the working human brain. Rev. Mod. Phys. 1993;65,413. https://doi.org/10.1103/revmodphys.65.413

[7] Annarumma L, D’Atri A, Alfonsi V, De Gennaro L. The Efficacy of Transcranial Current Stimulation Techniques to Modulate Resting-State EEG, to Affect Vigilance and to Promote Sleepiness. Brain Sci. 2018;8(7),137. https://doi.org/10.3390/brainsci8070137

[8] Utz KS, Dimova V, Oppenländer K, Kerkhoff G. Electrified minds: transcranial direct current stimulation (tDCS) and galvanic vestibular stimulation (GVS) as methods of noninvasive brain stimulation in neuropsychology — a review of current data and future implications. Neuropsychologia 2010;48(10),2789-2810. https://doi.org/10.1016/j.neuropsychologia.2010.06.002

[9] Lawson McLean A. Publication trends in transcranial magnetic stimulation: a 30-year panorama. Brain Stimulation 2019; in press. https://doi.org/10.1016/j.brs.2019.01.002

[10] Robinovitch LG. Electric analgesia, and electric resuscitation after heart failure under chloroform or electrocution. Journal of the American Medical Association 1911;LVI(7),478-481. https://doi.org/10.1001/jama.1911.02560070010003 
Gennaro L. Electrical stimulation of the frontal cortex enhances slow-frequency EEG activity and sleepiness. Neuroscience 2016;324,119-130.

https://doi.org/10.1016/j.neuroscience.2016.03.007

[12] Rosa, MA, Lisanby, SH. Somatic treatments for mood disorders.

Neuropsychopharmacology 2012; 37(1),102-16. 10.1038/npp.2011.225

[13] Helfrich RF, Schneider TR, Rach S, Trautmann-Lengsfeld SA, Engel A K, Herrmann CS. Entrainment of brain oscillations by transcranial alternating current stimulation. Current Biology: CB 2014;24(3),333-339.

https://doi.org/10.1016/j.cub.2013.12.041

[14] Gebodh N, Vacchi L, Adair D, Unal G, Poltorak A, Poltorak V, Bikson M. Proceedings \#11: Replay of Endogenous Sleep Rhythms to Produce Sleepiness. Brain Stimulation: Basic, Translational, and Clinical Research in Neuromodulation 2019; 12(2),e71-e72. https://doi.org/10.1016/j.brs.2018.12.180

[15] Schroeder CE, Wilson DA, Radman T, Scharfman H, Lakatos P. Dynamics of Active Sensing and perceptual selection. Current Opinion in Neurobiology 2010;20(2), 172-176. https://doi.org/10.1016/j.conb.2010.02.010

[16] Besle J, Schevon CA, Mehta AD, Lakatos P, Goodman RR, McKhann GM, Emerson RG, Schroeder, CE. Tuning of the human neocortex to the temporal dynamics of attended events. The Journal of Neuroscience: The Official Journal of the Society for Neuroscience 2011;31(9),3176-3185. https://doi.org/10.1523/JNEUROSCI.4518-10.2011

[17] Thomson JM, Goswami U. Rhythmic processing in children with developmental dyslexia: auditory and motor rhythms link to reading and spelling. Journal of Physiology, Paris 2008;102(1-3),120-129. https://doi.org/10.1016/j.jphysparis.2008.03.007 

in dyslexia: evidence for disruption to syllable timing. Hearing Research $2014 ; 308,141-$ 161. https://doi.org/10.1016/j.heares.2013.07.015

[19] Soltész F, Szücs D, Leong V, White S, Goswami U. Differential entrainment of neuroelectric delta oscillations in developmental dyslexia. PloS One 2013;8(10),e76608. https://doi.org/10.1371/journal.pone.0076608

[20] Lakatos P, Schroeder CE, Leitman DI, Javitt DC. Predictive suppression of cortical excitability and its deficit in schizophrenia. The Journal of Neuroscience: The Official Journal of the Society for Neuroscience 2013;33(28),11692-11702. https://doi.org/10.1523/JNEUROSCI.0010-13.2013

[21] Hanslmayr S, Matuschek J, Fellner M-C. Entrainment of Prefrontal Beta Oscillations Induces an Endogenous Echo and Impairs Memory Formation. Current Biology 2014;24(8),904-909. https://doi.org/10.1016/j.cub.2014.03.007

[22] Allen JJB, Keune PM, Schönenberg M, Nusslock R. Frontal EEG alpha asymmetry and emotion: From neural underpinnings and methodological considerations to psychopathology and social cognition. Psychophysiology 2018;55(1). https://doi.org/10.1111/psyp.13028

[23] Rogenmoser L, Zollinger N, Elmer S, Jäncke L. Independent component processes underlying emotions during natural music listening. Social Cognitive and Affective Neuroscience 2016;11(9),1428-1439. https://doi.org/10.1093/scan/nsw048

[24] Van der Vinne N, Vollebregt MA, van Putten MJAM, Arns M. Frontal alpha asymmetry as a diagnostic marker in depression: Fact or fiction? A meta-analysis. NeuroImage. Clinical 2017;16,79-87. https://doi.org/10.1016/j.nicl.2017.07.006 
M. Comorbid anxiety moderates the relationship between depression history and prefrontal EEG asymmetry. Psychophysiology 2018; 55(1).

https://doi.org/10.1111/psyp.12953

[26] Meyer T, Quaedflieg CWEM, Weijland K, Schruers K, Merckelbach H, Smeets T. Frontal EEG asymmetry during symptom provocation predicts subjective responses to intrusions in survivors with and without PTSD. Psychophysiology 2018;55(1). https://doi.org/10.1111/psyp.12779

[27] Chaieb L, Wilpert EC, Reber TP, Fell J. Auditory Beat Stimulation and its Effects on Cognition and Mood States. Frontiers in Psychiatry 2015;6.

https://doi.org/10.3389/fpsyt.2015.00070

[28] Kirsch DL, Nichols F. Cranial Electrotherapy Stimulation for Treatment of Anxiety, Depression, and Insomnia. Psychiatric Clinics of North America 2013;36(1), 169-176. https://doi.org/10.1016/j.psc.2013.01.006

[29] Rosenberg O, Shoenfeld N, Zangen A, Kotler M, Dannon PN. Deep TMS in a resistant major depressive disorder: a brief report. Depression and Anxiety 2010;27(5), 465-469. https://doi.org/10.1002/da.20689 


\section{Replicating Mental States}

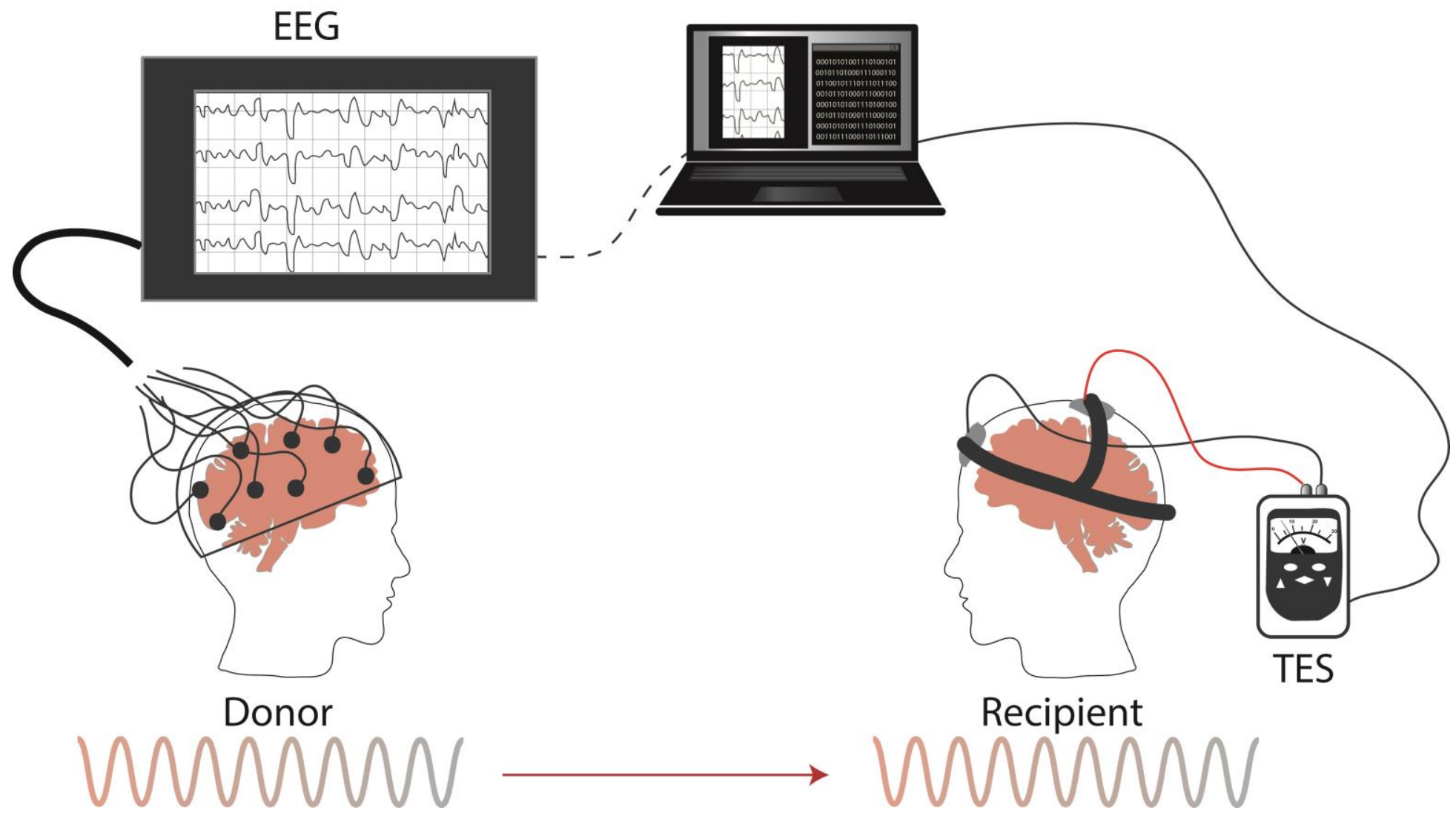

Figure 1 